\title{
RECENT RESULTS ON CLOSED G G $_{2}$ STRUCTURES
}

\author{
ANNA FINO AND ALBERTO RAFFERO
}

\begin{abstract}
We review recent results concerning closed $\mathrm{G}_{2}$-structures on seven-dimensional manifolds. In particular, we discuss the construction of examples and some related problems.
\end{abstract}

\section{INTRODUCTION}

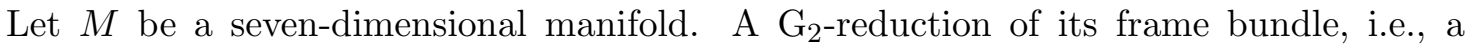
$\mathrm{G}_{2}$-structure, is characterised by the existence of a nowhere vanishing differential form $\varphi \in \Omega^{3}(M)$ with pointwise stabiliser isomorphic to the exceptional Lie group $\mathrm{G}_{2}$. Any such 3 -form $\varphi$ gives rise to a Riemannian metric $g_{\varphi}$ and to an orientation on $M$, and its covariant derivative $\nabla^{g_{\varphi}} \varphi$ with respect to the Levi Civita connection of $g_{\varphi}$ represents the obstruction to having $\operatorname{Hol}\left(g_{\varphi}\right) \subseteq \mathrm{G}_{2}$. By [17, a $\mathrm{G}_{2}$-structure $\varphi$ is parallel, i.e., $\nabla^{g_{\varphi}} \varphi=0$, if and only if both $d \varphi=0$ and $d *_{\varphi} \varphi=0$, where $*_{\varphi}$ is the Hodge operator associated with $g_{\varphi}$ and the given orientation. $\mathrm{A}_{2}$-structure satisfying the former condition is called closed, and a $\mathrm{G}_{2}$-structure satisfying the latter is called co-closed.

All known and potentially effective methods to obtain parallel $\mathrm{G}_{2}$-structures on compact 7-manifolds require the existence of a closed $\mathrm{G}_{2}$-structure, possibly satisfying some additional properties [3, 30, 34, 6, 35, 32, 41]. However, currently there are no known results guaranteeing the existence of closed $\mathrm{G}_{2}$-structures on compact manifolds, neither it is known whether the existence of this type of $\mathrm{G}_{2}$-structures imposes any constraint on the properties of the manifold, e.g. on its topology.

In this survey, we review various known examples of compact and non-compact 7-manifolds admitting closed $\mathrm{G}_{2}$-structures, and we discuss the techniques used to obtain them. Moreover, we give a new example satisfying some remarkable properties, and we present some related open problems.

\section{Preliminaries}

A $\mathrm{G}_{2}$-structure on a seven-dimensional manifold $M$ is a reduction of the structure group of its frame bundle to the exceptional Lie group $\mathrm{G}_{2}$. Any such reduction occurs if and only if $M$ is orientable and spin [27], and it is characterised by the existence of a 3 -form $\varphi \in \Omega^{3}(M)$ which can be written at each point $x$ of $M$ as

$$
\left.\varphi\right|_{x}=e^{127}+e^{347}+e^{567}+e^{135}-e^{146}-e^{236}-e^{245},
$$

with respect to some basis $\left\{e^{1}, \ldots, e^{7}\right\}$ of the cotangent space $T_{x}^{*} M$. Here and henceforth, the notation $e^{i j k \cdots}$ is used as a shorthand for the wedge product of covectors $e^{i} \wedge e^{j} \wedge e^{k} \wedge$ $\cdots$. Any differential 3 -form $\varphi$ defining a $\mathrm{G}_{2}$-structure is a section of an open subbundle 
$\Lambda_{+}^{3}\left(T^{*} M\right) \subset \Lambda^{3}\left(T^{*} M\right)$ with typical fibre isomorphic to $\mathrm{GL}(7, \mathbb{R}) / \mathrm{G}_{2}$. Therefore, small perturbations of $\varphi$ still define a $\mathrm{G}_{2}$-structure. We let $\Omega_{+}^{3}(M):=\Gamma\left(\Lambda_{+}^{3}\left(T^{*} M\right)\right)$.

Since $\mathrm{G}_{2} \subset \mathrm{SO}(7)$, every $\mathrm{G}_{2}$-structure $\varphi$ gives rise to a Riemannian metric $g_{\varphi}$ and to an orientation on $M$. In particular, $g_{\varphi}$ and the corresponding Riemannian volume form $d V_{\varphi}$ are related to $\varphi$ as follows

$$
g_{\varphi}(X, Y) d V_{\varphi}=\frac{1}{6} \iota_{X} \varphi \wedge \iota_{Y} \varphi \wedge \varphi
$$

for all $X, Y \in \Gamma(T M)$. We denote by $\nabla^{g_{\varphi}}$ the Levi Civita connection of $g_{\varphi}$, and by $*_{\varphi}$ the Hodge operator associated with $g_{\varphi}$ and the given orientation.

Given a $\mathrm{G}_{2}$-structure $\varphi \in \Omega_{+}^{3}(M)$, the obstruction to having $\operatorname{Hol}\left(g_{\varphi}\right) \subseteq \mathrm{G}_{2}$ is represented by the intrinsic torsion $T_{\varphi}$. The latter is a section of a vector bundle over $M$ with typical fibre $\left(\mathbb{R}^{7}\right)^{*} \otimes \mathfrak{g}_{2}^{\perp}$, where $\mathfrak{g}_{2}^{\perp}$ is the orthogonal complement in $\mathfrak{s o}(7)$ of the Lie algebra $\mathfrak{g}_{2}$ of $\mathrm{G}_{2}$. It is well-known that $T_{\varphi}$ can be identified with the covariant derivative $\nabla^{g_{\varphi}} \varphi$, so that one has $\operatorname{Hol}\left(g_{\varphi}\right) \subseteq \mathrm{G}_{2}$ if and only if $\nabla^{g_{\varphi}} \varphi=0$. When this happens, the $\mathrm{G}_{2}$-structure is said to be parallel or torsion-free. A further characterisation for parallel $\mathrm{G}_{2}$-structures was obtained by Fernández and Gray in [17]. We recall it in the following.

Theorem $2.1([17]) . A \mathrm{G}_{2}$-structure $\varphi$ is parallel if and only if both $d \varphi=0$ and $d *_{\varphi} \varphi=0$.

As proved by Bonan in [2], the metric induced by a parallel $\mathrm{G}_{2}$-structure is Ricci-flat. Thus, the construction of compact examples is not an easy task. For instance, compact homogeneous examples are exhausted by flat tori and, more generally, compact 7-manifolds with a parallel $\mathrm{G}_{2}$-structure $\varphi$ such that $\operatorname{Hol}\left(g_{\varphi}\right)=\mathrm{G}_{2}$ cannot have continuous symmetries. Indeed, using the Bochner-Weitzenböck technique, it is possible to show that the Lie algebra of the automorphism group $\operatorname{Aut}(M, \varphi)$ of a $\mathrm{G}_{2}$-structure $\varphi$

$$
\mathfrak{a u t}(M, \varphi):=\left\{X \in \Gamma(T M) \mid \mathcal{L}_{X} \varphi=0\right\} \subseteq \mathfrak{i s o m}\left(M, g_{\varphi}\right)
$$

is abelian with $\operatorname{dim}(\mathfrak{a} \mathfrak{u t}(M, \varphi))=0,1,3,7$, whenever $\varphi$ is parallel and $M$ is compact. The possible dimensions depend on having $\operatorname{Hol}\left(g_{\varphi}\right)=\mathrm{G}_{2}, \mathrm{SU}(3), \mathrm{SU}(2),\{1\}$, respectively.

Compact examples of Riemannian manifolds with holonomy equal to $\mathrm{G}_{2}$ were constructed in [30, 34, 6, 35, 32] using techniques such as orbifolds resolutions, generalised connected sums, and geometric glueing. All these manifolds admit a parallel $\mathrm{G}_{2}$-structure obtained by perturbing a $\mathrm{G}_{2}$-structure $\varphi$ satisfying the condition $d \varphi=0$ and whose intrinsic torsion is small in a suitable sense. The manifold topology plays a role in this construction, too, as the metric induced by a parallel $\mathrm{G}_{2}$-structure on a compact manifold $M$ has holonomy equal to $\mathrm{G}_{2}$ if and only if $\pi_{1}(M)$ is finite [30].

\section{Closed $\mathrm{G}_{2}$-Structures}

From Theorem 2.1, we know that a $\mathrm{G}_{2}$-structure $\varphi$ is parallel if and only if $d \varphi=0$ and $d *_{\varphi} \varphi=0$. $\mathrm{A} \mathrm{G}_{2}$-structure satisfying the former condition is called closed, and one satisfying the latter is called co-closed. As we have previously recalled, closed $\mathrm{G}_{2}$-structures play a central role in the known constructions of compact 7-manifolds with holonomy $\mathrm{G}_{2}$. However, currently there are no general results ensuring the existence of closed $\mathrm{G}_{2}$-structures on compact manifolds, neither it is known whether the existence of such type of structure imposes any constraint on the manifold structure, e.g. on its topology. On the other hand, 
Crowley and Nordström proved that the existence of co-closed $\mathrm{G}_{2}$-structures on compact 7-manifolds is only a topological matter. Indeed, they always exist on any compact manifold admitting $\mathrm{G}_{2}$-structures [9].

By [28, a closed $\mathrm{G}_{2}$-structure $\varphi \in \Omega_{+}^{3}(M)$ defines a calibration on $M$, that is, for every oriented tangent 3-plane $V \subset T_{x} M$ one has $\left.\varphi\right|_{V} \leq \operatorname{vol}_{V}$, where $\operatorname{vol}_{V}$ denotes the volume form of the inner product $\left.g_{\varphi}\right|_{V}$ on $V$. A three-dimensional oriented submanifold $N \hookrightarrow M$ is said to be associative if it is calibrated by $\varphi$, i.e., if $\left.\varphi\right|_{T_{x} N}=\operatorname{vol}_{T_{x} N}$ for all $x \in N$. Recall that every compact associative 3 -fold is volume minimising in its homology class (see [28, Thm. II.4.2]). Similarly, if a $\mathrm{G}_{2}$-structure $\varphi$ is co-closed, then the 4-form $*_{\varphi} \varphi$ defines a calibration on $M$, and one can consider four-dimensional oriented submanifolds of $M$ calibrated by it. These submanifolds are called co-associative. It is possible to show that a four-dimensional oriented submanifold $U$ in $M$ is co-associative if and only if $\left.\varphi\right|_{U} \equiv 0$ (cf. [28, Cor. IV.1.20]). Thus, when a $\mathrm{G}_{2}$-structure $\varphi$ is closed but not co-closed, one can define an oriented 4-fold $U$ of $M$ to be co-associative if $\left.\varphi\right|_{U} \equiv 0$. However, $U$ is not necessarily volume minimising in its homology class, as $d *_{\varphi} \varphi \neq 0$. For more details on associative and co-associative submanifolds, we refer the reader to [28, 31].

Let us now focus on a 7-manifold $M$ endowed with a closed $\mathrm{G}_{2}$-structure $\varphi$. In this case, the intrinsic torsion $T_{\varphi}$ can be identified with a unique 2-form $\tau \in \Omega_{14}^{2}(M):=$ $\left\{\alpha \in \Omega^{2}(M) \mid \alpha \wedge \varphi=-*_{\varphi} \alpha\right\}$ such that

$$
d *_{\varphi} \varphi=\tau \wedge \varphi
$$

We shall refer to $\tau$ as the intrinsic torsion form of $\varphi$. Notice that $\tau$ vanishes identically if and only if $d *_{\varphi} \varphi=0$, that is, if and only if the closed $\mathrm{G}_{2}$-structure is parallel.

The Ricci tensor and the scalar curvature of the Riemannian metric $g_{\varphi}$ induced by $\varphi$ can be expressed in terms of the intrinsic torsion form $\tau$ as follows (cf. [3]):

$$
\begin{aligned}
\operatorname{Ric}\left(g_{\varphi}\right) & =\frac{1}{4}|\tau|^{2} g_{\varphi}-\frac{1}{4} j_{\varphi}\left(d \tau-\frac{1}{2} *_{\varphi}(\tau \wedge \tau)\right), \\
\operatorname{Scal}\left(g_{\varphi}\right) & =-\frac{1}{2}|\tau|^{2},
\end{aligned}
$$

where the map $j_{\varphi}: \Omega^{3}(M) \rightarrow \mathcal{S}^{2}(M)$ assigns to each 3 -form $\gamma$ the symmetric 2-covariant tensor $j_{\varphi}(\gamma)(X, Y)=*_{\varphi}\left(\iota_{X} \varphi \wedge \iota_{Y} \varphi \wedge \gamma\right)$. In particular, the scalar curvature is always nonpositive, and it vanishes identically if and only if $\varphi$ is parallel. Further properties of $\operatorname{Ric}\left(g_{\varphi}\right)$ and $\operatorname{Scal}\left(g_{\varphi}\right)$ when $M$ is compact are summarised in the next theorem.

Theorem 3.1 ([3, 7]). Let $M$ be a compact 7-manifold endowed with a closed $\mathrm{G}_{2}$-structure $\varphi$. Then,

(1) $g_{\varphi}$ is Einstein if and only if $\varphi$ is parallel;

(2) $\int_{M}\left[\operatorname{Scal}\left(g_{\varphi}\right)\right]^{2} d V_{\varphi} \leq 3 \int_{M}\left|\operatorname{Ric}\left(g_{\varphi}\right)\right|^{2} d V_{\varphi}$.

Remark 3.2. Currently, it is still not known whether there exist (even incomplete) closed non-parallel $\mathrm{G}_{2}$-structures inducing an Einstein metric. A negative answer when the 7manifold is a simply connected solvable Lie group endowed with a left-invariant closed $\mathrm{G}_{2}$-structure was given in [14]. 
In [3, Bryant proved that the inequality in point 2, of Theorem 3.1 reduces to an equality if and only if

$$
d \tau=\frac{|\tau|^{2}}{6} \varphi+\frac{1}{6} *_{\varphi}(\tau \wedge \tau) .
$$

A closed $\mathrm{G}_{2}$-structure $\varphi$ satisfying the above equation is called extremally Ricci pinched ( $E R P$ for short). Notice that this definition makes sense also in the non-compact setting.

The existence of an ERP $\mathrm{G}_{2}$-structure on a compact 7-manifold imposes strong constraints on its geometry. In detail:

Proposition $3.3([3])$. Let $M$ be a compact 7-manifold endowed with an ERP $\mathrm{G}_{2}$-structure $\varphi$ with intrinsic torsion form $\tau \in \Omega_{14}^{2}(M)$ not identically vanishing. Then

(1) $\tau$ has constant (non-zero) norm and $\tau \wedge \tau \wedge \tau=0$;

(2) $\tau \wedge \tau$ and $*_{\varphi}(\tau \wedge \tau)$ are non-zero closed simple forms of constant norm. Consequently, the tangent bundle of $M$ splits into the orthogonal direct sum of two integrable subbundles $T M=P \oplus Q$, with $P:=\operatorname{ker}(\tau \wedge \tau), Q:=\operatorname{ker}\left(*_{\varphi}(\tau \wedge \tau)\right)$. Moreover, the P-leaves are associative submanifolds calibrated by $-|\tau|^{-2} *_{\varphi}(\tau \wedge \tau)$, and the $Q$-leaves are coassociative submanifolds calibrated by $-|\tau|^{-2}(\tau \wedge \tau)$;

(3) the Ricci tensor of $g_{\varphi}$ is given by $\operatorname{Ric}\left(g_{\varphi}\right)=\frac{1}{12} j_{\varphi}\left(*_{\varphi}(\tau \wedge \tau)\right)=-\left.\frac{1}{6}|\tau|^{2} g_{\varphi}\right|_{P}$. Hence, it is non-positive with eigenvalues $-\frac{1}{6}|\tau|^{2}$ of multiplicity three and 0 of multiplicity four.

So far, the only known compact examples were obtained in 3,33 . The example in [3] consists of the quotient $\Gamma \backslash M$, where $M=\mathrm{SL}(2, \mathbb{C}) \ltimes \mathbb{C}^{2} / \mathrm{SU}(2)$ is a non-compact ho-

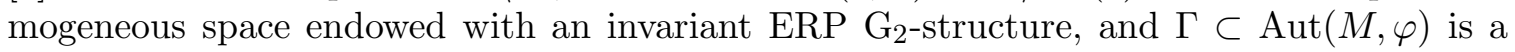
co-compact discrete subgroup. The example in [33] is the compact quotient of a simply connected solvable Lie group endowed with the left-invariant ERP $\mathrm{G}_{2}$-structure obtained in [37, Ex. 4.7]. Further examples are given in [1, 24, 38, 39].

\section{Examples of manifolds admitting Closed $\mathrm{G}_{2}$-STRuCtures}

Since there are no general results ensuring the existence of closed $\mathrm{G}_{2}$-structures on compact 7-manifolds, one should in principle try to solve the PDEs arising from the equation $d \varphi=0$ for a generic 3 -form $\varphi \in \Omega_{+}^{3}(M)$ on a given compact manifold $M$. In some cases, this problem may be reduced to a simpler one. In this section, we give an overview of various known examples and we discuss some new ones.

4.1. Closed $\mathbf{G}_{\mathbf{2}}$-structures with symmetry. The first examples of compact 7-manifolds admitting closed non-parallel $\mathrm{G}_{2}$-structures were obtained by Fernández in [11, 12. These examples are of the form $M=\Gamma \backslash \mathrm{G}$, where $\mathrm{G}$ is a seven-dimensional simply connected nilpotent or solvable Lie group, $\Gamma \subset \mathrm{G}$ is a co-compact discrete subgroup (lattice), and the closed $\mathrm{G}_{2}$-structure on $M$ is induced by a left-invariant one on $\mathrm{G}$. In particular, $(M, \varphi)$ is locally homogeneous. Further examples of this type have been obtained in [5, 26, 36]. We emphasise that in all these examples the Lie group $G$, being diffeomorphic to $\mathbb{R}^{7}$, is not compact. Moreover, any left-invariant $\mathrm{G}_{2}$-structure $\varphi$ on $\mathrm{G}$ is completely determined by the 3 -form $\left.\varphi\right|_{1_{\mathrm{G}}}$ on the Lie algebra $\mathfrak{g} \cong T_{1_{\mathrm{G}}} \mathrm{G}$, and the condition $d \varphi=0$ is equivalent to a system of algebraic equations for the coefficients of $\left.\varphi\right|_{1_{\mathrm{G}}}$. 
As this last observation suggests, symmetry considerations might help to reduce the PDEs arising from the condition $d \varphi=0$ to equations of a simpler type. Therefore, one may ask whether there exist examples of compact 7-manifolds $M$ endowed with a closed non-parallel $\mathrm{G}_{2}$-structure $\varphi$ that are acted on with low cohomogeneity by the automorphism group $\operatorname{Aut}(M, \varphi)$ or a subgroup thereof. In [8], Cleyton and Swann considered 7-manifolds endowed with a $\mathrm{G}_{2}$-structure and acted on with cohomogeneity one by a simple group of automorphisms, showing in particular that if $\varphi$ is closed then only complete non-compact examples occur. Moreover, the existence of compact homogeneous 7-manifolds endowed with an invariant closed non-parallel $\mathrm{G}_{2}$-structure, i.e., having a transitive group of automorphisms $\mathrm{G} \subset \operatorname{Aut}(M, \varphi)$, has been open for some time (see [37, Question 3.1]).

In [43, Podestà and the second named author of the present survey studied the properties of $\operatorname{Aut}(M, \varphi)$ when $M$ is compact and the $\mathrm{G}_{2}$-structure $\varphi$ is closed and non-parallel, obtaining the following result.

Theorem 4.1 (43]). Let $M$ be a compact 7-manifold endowed with a closed non-parallel $\mathrm{G}_{2}$-structure $\varphi$. Then, $\mathfrak{a} \mathfrak{u}(M, \varphi)$ is abelian with dimension bounded by $\min \left\{6, b_{2}(M)\right\}$.

The above result immediately implies that there are no compact homogeneous 7 -manifolds endowed with an invariant closed non-parallel $\mathrm{G}_{2}$-structure, and that the only cohomogeneity one examples occur on manifolds diffeomorphic to the 7-torus.

Notice that the examples of seven-dimensional simply connected Lie groups $\mathrm{G}$ endowed with a left-invariant closed $\mathrm{G}_{2}$-structure $\varphi$ mentioned earlier $([11,12$, 5, 26, 36]) correspond to the case when $M$ is not compact and $\mathrm{G} \subset \operatorname{Aut}(M, \varphi)$ acts simply transitively on it. Recently, the complete classification of non-compact 7-manifolds admitting closed nonparallel $\mathrm{G}_{2}$-structures and acted on transitively by a reductive group of automorphisms has been obtained in [46].

We remark that Theorem 4.1 gives some insights on a currently open problem concerning the existence of a closed $\mathrm{G}_{2}$-structure on the 7 -sphere $\mathbb{S}^{7}$, as it implies that $\operatorname{Aut}\left(\mathbb{S}^{7}, \varphi\right)$ must be finite for any hypothetical closed $\mathrm{G}_{2}$-structure $\varphi \in \Omega_{+}^{3}\left(\mathbb{S}^{7}\right)$.

4.2. A compact example obtained via the resolution of an orbifold. Besides symmetry considerations, other techniques may be used to construct examples of compact 7-manifolds admitting closed $\mathrm{G}_{2}$-structures. For instance, all known examples of compact manifolds admitting parallel $\mathrm{G}_{2}$-structures are endowed with a closed one. The techniques used to obtain these examples include orbifolds resolutions [30, the twisted connected sum construction [34, 6, 35], and analysis on families of Eguchi-Hanson spaces [32].

Recently, Fernández, Kovalev, Muñoz and the first named author of the present survey obtained a new example of a compact 7-manifold $M$ with $b_{1}(M)=1$ and admitting closed $\mathrm{G}_{2}$-structures but no parallel $\mathrm{G}_{2}$-structures [13]. This example is constructed resolving the singularities of a certain orbifold, and the techniques involved in this construction are motivated by those used by Joyce to obtain compact 7 -manifolds with holonomy $\mathrm{G}_{2}$ via the resolution of orbifolds of the form $\mathbb{T}^{7} / \mathrm{F}$, where $\mathrm{F} \subset \mathrm{G}_{2}$ is a suitable finite subgroup [30].

The idea of [13] consists in starting with a compact nilmanifold $M=\Gamma \backslash \mathrm{N}$ endowed with an invariant closed $G_{2}$-structure in place of the 7 -torus $\mathbb{T}^{7} \cong \mathbb{R}^{7} / \mathbb{Z}^{7}$ endowed with the flat $\mathrm{G}_{2}$-structure induced by the canonical one on $\mathbb{R}^{7}$. Here, $\mathrm{N}$ is the simply-connected nilpotent Lie group corresponding to the 3 -step nilpotent Lie algebra $\mathfrak{n}=\left\langle e_{1}, \ldots, e_{7}\right\rangle$ with 
the following structure equations

$$
\left[e_{1}, e_{2}\right]=-e_{4},\left[e_{1}, e_{3}\right]=-e_{5},\left[e_{1}, e_{4}\right]=-e_{6},\left[e_{1}, e_{5}\right]=-e_{7},
$$

$\Gamma \cong 2 \mathbb{Z} \times \mathbb{Z}^{6}$ is a lattice in $\mathrm{N}$, and the invariant closed $\mathrm{G}_{2}$-structure on $M$ is induced by a left-invariant one on $\mathrm{N}$. One then considers the action of the finite group $\mathrm{F}=\mathbb{Z}_{2}$ on $\mathrm{N}$ generated by

$$
\rho: \mathrm{N} \rightarrow \mathrm{N}, \quad\left(x_{1}, \ldots, x_{7}\right) \mapsto\left(-x_{1},-x_{2}, x_{3}, x_{4},-x_{5},-x_{6}, x_{7}\right) .
$$

This action satisfies $\rho(a b)=\rho(a) \rho(b)$, for all $a, b \in \mathrm{N}$, and $\rho(\Gamma)=\Gamma$. Thus, $\rho$ induces an action of $\mathbb{Z}_{2}$ on $M=\Gamma \backslash \mathrm{N}$, and it is possible to consider the quotient space $\widehat{M}=M / \mathbb{Z}_{2}$. The following result can then be proved.

Theorem $4.2([13)$.

- $\widehat{M}=M / \mathbb{Z}_{2}$ is a compact 7-orbifold with first Betti number $b_{1}(\widehat{M})=1$ and admitting an orbifold closed $\mathrm{G}_{2}$-form $\widehat{\varphi}$. The singular locus $S$ of $\widehat{M}$ is the disjoint union of 16 copies of $\mathbb{T}^{3}$.

- There exists a resolution $\pi:(\widetilde{M}, \widetilde{\varphi}) \rightarrow(\widehat{M}, \widehat{\varphi})$, where $\widetilde{M}$ is a compact smooth manifold, $b_{1}(\widetilde{M})=1$, and $\widetilde{\varphi}=\pi^{*} \widehat{\varphi}$ in the complement of a neighborhood of the exceptional locus $\pi^{-1}(S)$. Moreover, $\pi_{1}(\widetilde{M})=\mathbb{Z}, \widetilde{M}$ is formal and it does not admit any parallel $\mathrm{G}_{2}$ structure.

4.3. A comparison with Lie algebras admitting symplectic structures. As we recalled in Section 4.1, there are many (non-compact) examples of simply connected sevendimensional Lie groups admitting left-invariant closed $\mathrm{G}_{2}$-structures, and for any such example the properties of the $\mathrm{G}_{2}$-structure may be studied at the Lie algebra level. This leads one to focusing on seven-dimensional Lie algebras $\mathfrak{g}$ endowed with a $\mathrm{G}_{2}$-structure $\varphi \in \Lambda_{+}^{3}\left(\mathfrak{g}^{*}\right)$ which is closed with respect to the Chevalley-Eilenberg differential $d$ of $\mathfrak{g}$. In this setting, there are some problems motivated by symplectic geometry that it is worth investigating.

It is known that symplectic structures and closed $\mathrm{G}_{2}$-structures share similar properties. Indeed, they are both defined by closed differential forms satisfying the same non-degeneracy condition, namely at each point of the manifold their orbit under the action of the general linear group is open (see [29]). Moreover, the existence of a symplectic structure on an even-dimensional Lie algebra imposes some constraints on it. For instance, a unimodular Lie algebra admitting a symplectic structure must be solvable [4, 40], and any unimodular Lie algebra cannot admit symplectic structures defined by an exact 2 -form [10]. Thus, it is natural to investigate whether similar results hold for seven-dimensional unimodular Lie algebras admitting closed $\mathrm{G}_{2}$-structures, too. In our works [16, 22], we obtained a negative answer to these problems.

In [22], we studied the existence of closed $\mathrm{G}_{2}$-structures on non-solvable unimodular Lie algebras, showing that examples actually occur and obtaining a complete classification of the possible Lie algebras up to isomorphism.

Theorem 4.3 ([22]). Let $\mathfrak{g}$ be a seven-dimensional non-solvable unimodular Lie algebra admitting closed $\mathrm{G}_{2}$-structures, and denote by $\mathfrak{g}=\mathfrak{s} \ltimes \mathfrak{r}$ its Levi decomposition, where $\mathfrak{r}$ is the radical of $\mathfrak{g}$ and $\mathfrak{s}$ is a semisimple subalgebra. Then, $\mathfrak{s} \cong \mathfrak{s l}(2, \mathbb{R})$ and 
- if the Levi decomposition is trivial, namely $\mathfrak{g}=\mathfrak{s} \oplus \mathfrak{r}$, then $\mathfrak{r}$ is centerless, and there exists a basis $\left\{e^{1}, \ldots, e^{7}\right\}$ of $\mathfrak{g}^{*}$ for which its structure equations $\left(d e^{1}, \ldots, d e^{7}\right)$ are in the following list

$$
\begin{aligned}
& \left(-e^{23},-2 e^{12}, 2 e^{13}, 0,-e^{45}, \frac{1}{2} e^{46},-e^{47}, \frac{1}{2} e^{47}\right) \\
& \left(-e^{23},-2 e^{12}, 2 e^{13}, 0,-e^{45},-\mu e^{46},(1+\mu) e^{47}\right),-1<\mu \leq \frac{1}{2} \\
& \left(-e^{23},-2 e^{12}, 2 e^{13}, 0,-\mu e^{45}, \frac{\mu}{2} e^{46}-e^{47}, e^{46}+\frac{\mu}{2} e^{47}\right), \mu>0 .
\end{aligned}
$$

- if the Levi decomposition is not trivial, then $\mathfrak{r} \cong \mathbb{R} \ltimes \mathbb{R}^{3}$, and there is a basis $\left\{e^{1}, \ldots, e^{7}\right\}$ of $\mathfrak{g}^{*}$ for which its structure equations are the following

$$
\left(-e^{23},-2 e^{12}, 2 e^{13},-e^{14}-e^{25}-e^{47}, e^{15}-e^{34}-e^{57}, 2 e^{67}, 0\right) .
$$

In our recent joint work with M. Fernández [16], we focused on closed $\mathrm{G}_{2}$-structures defined by an exact 3 -form $\varphi$ on a unimodular Lie algebra $\mathfrak{g}$, showing that there exist examples when the third Betti number $b_{3}(\mathfrak{g})$ of $\mathfrak{g}$ vanishes and when $\mathfrak{g}$ is $(2,3)$-trivial, i.e., when $b_{2}(\mathfrak{g})=0=b_{3}(\mathfrak{g})$.

4.4. Products, warped products, and Lie algebras extensions. Further examples not mentioned so far can be obtained on products of suitable manifolds. For instance, it is possible to define closed $\mathrm{G}_{2}$-structures on the product of a 4-manifold endowed with a hypersymplectic structure and the 3 -torus $\mathbb{T}^{3}$ (see e.g. [19]), and on the product of a 6 -manifold endowed with a suitable $\mathrm{SU}(3)$-structure and the circle $\mathbb{S}^{1}$.

Recall that an $\mathrm{SU}(3)$-structure on a 6 -manifold $N$ is the data of an almost Hermitian structure $(g, J)$, with corresponding fundamental 2-form $\omega:=g(J \cdot, \cdot)$, and a complex $(3,0)$ form $\Psi=\psi+i \widehat{\psi}$ satisfying the normalisation condition $3 \psi \wedge \widehat{\psi}=2 \omega^{3}$. Moreover, the whole structure is completely determined by the real 2-form $\omega$ and the real 3-form $\psi$, provided that they satisfy suitable conditions (see [29] for details). Given a positive function $f \in \mathcal{C}^{\infty}(N)$, it is possible to endow the product $N \times \mathbb{S}^{1}$ with the 3 -form

$$
\varphi=f \omega \wedge d s+\psi
$$

which is easily seen to define a $\mathrm{G}_{2}$-structure with associated Riemannian metric $g_{\varphi}=$ $g+f^{2} d s^{2}$, where $s$ denotes the coordinate on the circle. Notice that the manifold $N \times \mathbb{S}^{1}$ endowed with this metric is none other than the warped product of $N$ and $\mathbb{S}^{1}$ with warping function $f$ and fibre $\mathbb{S}^{1}$. The $\mathrm{G}_{2}$-structure $\varphi$ on $N \times \mathbb{S}^{1}$ is closed if and only if the $\mathrm{SU}(3)$ structure on $N$ satisfies

$$
d \omega=-d \log (f) \wedge \omega, \quad d \psi=0 .
$$

Thus, the problem of finding a closed $\mathrm{G}_{2}$-structure of the form (4.1) on $N \times \mathbb{S}^{1}$ boils down to the construction of a 6 -manifold endowed with an $\mathrm{SU}(3)$-structure satisfying (4.2). An example on $N=\mathbb{T}^{6}$ can be found in 23 .

When the warping function $f$ is constant, the manifold reduces to a Riemannian product, and it is always possible to assume that $f \equiv 1$ up to scaling the coordinate $s$. In this case, the $\mathrm{G}_{2}$-structure $\varphi=\omega \wedge d s+\psi$ is closed if and only if both $d \omega=0$ and $d \psi=0$. An $\mathrm{SU}(3)$-structure satisfying these conditions is called symplectic half-flat, and its intrinsic torsion can be identified with the unique real 2-form $w_{2}$ of type $(1,1)$ such that $w_{2} \wedge \omega^{2}=0$ and $d \widehat{\psi}=w_{2} \wedge \omega$. A simple computation shows that the intrinsic torsion form of the closed 
$\mathrm{G}_{2}$-structure $\varphi=\omega \wedge d s+\psi$ coincides with $w_{2}$. We refer the reader to [18, 23, 44, 45] and the references therein for more details and examples.

The last example of closed $\mathrm{G}_{2}$-structures on $N \times \mathbb{S}^{1}$ that we would like to mention is obtained as follows. Let us consider a coupled SU(3)-structure on $N$, i.e., an $\mathrm{SU}(3)$-structure satisfying the condition $d \omega=c \psi$ for some non-zero real number $c$. The 3 -form $\psi$ is clearly closed, while the 3 -form $\widehat{\psi}$ satisfies

$$
d \widehat{\psi}=-\frac{2}{3} c \omega^{2}+w_{2} \wedge \omega
$$

for a unique real 2 -form $w_{2}$ of type $(1,1)$ and satisfying $w_{2} \wedge \omega^{2}=0$. The pair $\left(c, w_{2}\right)$ completely determines the intrinsic torsion of the coupled $\mathrm{SU}(3)$-structure $(\omega, \psi)$. Now, the $\mathrm{G}_{2}$-structure $\varphi=\omega \wedge d s+\psi$ on $N \times \mathbb{S}^{1}$ considered before is no longer closed, but there exists a global conformal change making it into a closed one, namely

$$
\widetilde{\varphi}=e^{c s}(\omega \wedge d s+\psi) \text {. }
$$

The Riemannian metric induced by $\widetilde{\varphi}$ is given by $g_{\widetilde{\varphi}}=e^{\frac{2}{3} c s} g_{\varphi}$, and its intrinsic torsion form is $\widetilde{\tau}=e^{\frac{c s}{3}} w_{2}$. For more details and examples regarding coupled $\mathrm{SU}(3)$-structures and $\mathrm{G}_{2}$-structures that are (locally) conformal to closed ones, the reader may refer to [15, 21].

As for Lie algebras, similar constructions can be made considering rank-one extensions of six-dimensional Lie algebras endowed with an $\mathrm{SU}(3)$-structure.

We recall that the rank-one extension of an $n$-dimensional Lie algebra $\mathfrak{h}$ induced by a derivation $D \in \operatorname{Der}(\mathfrak{h})$ is the $(n+1)$-dimensional Lie algebra given by the vector space $\mathfrak{h} \oplus \mathbb{R}$ endowed with the Lie bracket

$$
[(X, a),(Y, b)]:=\left([X, Y]_{\mathfrak{h}}+a D(Y)-b D(X), 0\right),
$$

where $(X, a),(Y, b) \in \mathfrak{h} \oplus \mathbb{R}$, and $[\cdot, \cdot]_{\mathfrak{h}}$ is the Lie bracket on $\mathfrak{h}$. We shall denote this Lie algebra by $\mathfrak{h} \rtimes_{D} \mathbb{R}$. Moreover, we let $\xi:=(0,1)$, and we denote by $\eta$ the 1 -form on $\mathfrak{h} \rtimes_{D} \mathbb{R}$ such that $\eta(\xi)=1$ and $\mathfrak{h}=\operatorname{ker}(\eta)$.

Let $d$ and $\hat{d}$ denote the Chevalley-Eilenberg differential on $\mathfrak{h} \rtimes_{D} \mathbb{R}$ and on $\mathfrak{h}$, respectively. Then, for every $k$-form $\gamma \in \Lambda^{k}\left(\mathfrak{h}^{*}\right)$ the following identity holds:

$$
d \gamma=\hat{d} \gamma+(-1)^{k+1} D^{*} \gamma \wedge \eta
$$

where the natural action of an endomorphism $A \in \operatorname{End}(\mathfrak{h})$ on $\Lambda^{k}\left(\mathfrak{h}^{*}\right)$ is given by

$$
A^{*} \gamma\left(X_{1}, \ldots, X_{k}\right)=\gamma\left(A X_{1}, \ldots, X_{k}\right)+\cdots+\gamma\left(X_{1}, \ldots, A X_{k}\right),
$$

for all $X_{1}, \ldots, X_{k} \in \mathfrak{h}$. If $D=0 \in \operatorname{Der}(\mathfrak{h})$ is the trivial derivation, then the rank-one extension of $\mathfrak{h}$ reduces to the product Lie algebra $\mathfrak{h} \oplus \mathbb{R}$.

Assume now that $\mathfrak{h}$ is six-dimensional and that it is endowed with an $\mathrm{SU}(3)$-structure $(\omega, \psi)$. Then, the rank-one extension $\mathfrak{h} \rtimes_{D} \mathbb{R}$ is always endowed with a $\mathrm{G}_{2}$-structure defined by the 3 -form $\varphi=\omega \wedge \eta+\psi$. Clearly, the condition $d \varphi=0$ constrains the possible types of $\mathrm{SU}(3)$-structures and the properties of the derivation $D$ that one can consider. Indeed, it holds if and only if

$$
\hat{d} \omega=-D^{*} \psi, \quad \hat{d} \psi=0
$$


In the next proposition, whose proof immediately follows from (4.4), we recall some relevant cases related to the $\mathrm{SU}(3)$-structures mentioned above.

Proposition 4.4. Let $\mathfrak{h}$ be a six-dimensional Lie algebra endowed with an $\mathrm{SU}(3)$-structure $(\omega, \psi)$, and consider the rank-one extension $\mathfrak{h} \rtimes_{D} \mathbb{R}$ endowed with the $\mathrm{G}_{2}$-structure $\varphi=$ $\omega \wedge \eta+\psi$. Then, $\varphi$ is closed in the following cases

- the $\mathrm{SU}(3)$-structure is symplectic half-flat, i.e., $\hat{d} \omega=0, \hat{d} \psi=0$, and either $D=0$ or $D^{*} \psi=0$

- the $\mathrm{SU}(3)$-structure is coupled with $\hat{d} \omega=c \psi$, and $D^{*} \psi=-c \psi$.

The classification of six-dimensional solvable Lie algebras admitting symplectic half-flat $\mathrm{SU}(3)$-structures was given in [18. Examples of closed $\mathrm{G}_{2}$-structures on rank-one extensions of these Lie algebras are discussed in 42 .

In [21], we proved that a six-dimensional nilpotent Lie algebra admitting coupled SU(3)structures is isomorphic to one of the following

$$
\mathfrak{n}_{1}=\left(0,0,0, e^{13}, e^{14}+e^{23}, e^{13}-e^{15}-e^{24}\right), \quad \mathfrak{n}_{2}=\left(0,0,0,0, e^{14}+e^{23}, e^{13}-e^{24}\right) .
$$

In both cases, there exists a coupled $\mathrm{SU}(3)$-structure with adapted basis $\left\{e^{1}, \ldots, e^{6}\right\}$, namely

$$
\omega=e^{12}+e^{34}+e^{56}, \quad \Psi=\left(e^{1}+i e^{2}\right) \wedge\left(e^{3}+i e^{4}\right) \wedge\left(e^{5}+i e^{6}\right)
$$

and it is easy to check that $\hat{d} \omega=-\psi$, i.e., $c=-1$. Moreover, for the coupled SU(3)structure on $\mathfrak{n}_{2}$, the unique 2 -form $w_{2}$ fulfilling (4.3) is given by $w_{2}=\frac{4}{3} e^{12}+\frac{4}{3} e^{34}-\frac{8}{3} e^{56}$ and its differential $\hat{d} w_{2}$ is proportional to $\psi$.

Remark 4.5. More generally, if $(\omega, \psi)$ is a coupled SU(3)-structure on a 6-manifold with $d w_{2}$ proportional to $\psi$, then one has $d w_{2}=\frac{\left|w_{2}\right|^{2}}{4} \psi$ with $\left|w_{2}\right|$ constant (see e.g. [20]). Furthermore, on the nilpotent Lie algebra $\mathfrak{n}_{1}$ there are no coupled SU(3)-structures satisfying this condition (cf. [20, Prop. 12]).

The nilpotent Lie algebra $\mathfrak{n}_{2}$ endowed with the coupled $\mathrm{SU}(3)$-structure $(\omega, \psi)$ given in (4.5) admits rank-one extensions for which the $\mathrm{G}_{2}$-structure $\varphi=\omega \wedge \eta+\psi$ is closed. A one-parameter family of examples was given by Lauret in [37, Ex. 4.10], and it consists of the extension $\mathfrak{g}_{a}:=\mathfrak{n}_{2} \rtimes_{D_{a}} \mathbb{R}$ with respect to the derivation

$$
D_{a}=\operatorname{diag}\left(a, a, \frac{1}{2}-a, \frac{1}{2}-a, \frac{1}{2}, \frac{1}{2}\right) \in \operatorname{Der}\left(\mathfrak{n}_{2}\right), \quad a \geq \frac{1}{4} .
$$

Notice that $D_{a}$ fulfills the condition $D_{a}^{*} \psi=\psi$, whence $d \varphi=0$. The Lie algebra $\mathfrak{g}_{a}$ is completely solvable, and the bound on the real parameter $a$ arises requiring the simply connected Lie groups $\mathrm{G}_{a}$ with Lie algebra $\mathfrak{g}_{a}$ to be pairwise non-isomorphic for different values of $a$.

The Lie algebra $\mathfrak{n}_{1}$ endowed with the coupled $\mathrm{SU}(3)$-structure given in (4.5) admits derivations satisfying the condition $D^{*} \psi=\psi$, too. It is not difficult to prove that the most 
general one has the following expression with respect to the basis $\left\{e_{1}, \ldots, e_{6}\right\}$ of $\mathfrak{n}_{1}$

$$
D_{a, b}=\left(\begin{array}{cccccc}
0 & 0 & 0 & 0 & 0 & 0 \\
0 & 0 & 0 & 0 & 0 & 0 \\
a & 0 & \frac{1}{2} & 0 & 0 & 0 \\
0 & a & 0 & \frac{1}{2} & 0 & 0 \\
b & 0 & 0 & 0 & \frac{1}{2} & 0 \\
0 & b & 0 & 0 & 0 & \frac{1}{2}
\end{array}\right), \quad a, b \in \mathbb{R}
$$

Consequently, any Lie algebra belonging to the two-parameter family $\mathfrak{g}_{a, b}:=\mathfrak{n}_{1} \rtimes_{D_{a, b}} \mathbb{R}$ admits a closed $\mathrm{G}_{2}$-structure defined by $\varphi=\omega \wedge \eta+\psi$.

We now discuss a new example of a solvable non-nilpotent Lie algebra endowed with a coupled SU(3)-structure, and we show that there exist rank-one extensions admitting closed $\mathrm{G}_{2}$-structures.

Example 4.6. Consider the two parameter family of six-dimensional unimodular solvable Lie algebras $\mathfrak{s}_{a, b}, a, b \in \mathbb{R}$, whose structure equations with respect to a basis $\left\{e^{1}, \ldots, e^{6}\right\}$ of $\mathfrak{s}_{a, b}^{*}$ are the following

$$
\left\{\begin{array}{l}
d e^{1}=-a e^{26} \\
d e^{2}=a e^{16} \\
d e^{3}=b e^{16}+b e^{25}+a e^{46} \\
d e^{4}=b e^{15}-b e^{26}-a e^{36} \\
d e^{5}=d e^{6}=0
\end{array}\right.
$$

Notice that $\mathfrak{s}_{a, b} \cong \mathbb{R}^{4} \rtimes \mathbb{R}^{2}$, where the subalgebra $\mathbb{R}^{2}$ is spanned by the vectors $e_{5}$ and $e_{6}$. We endow $\mathfrak{s}_{a, b}$ with the $\mathrm{SU}(3)$-structure $(\omega, \psi)$ given by (4.5).

If $a=0$, then the Lie algebra $\mathfrak{s}_{0, b}$ is isomorphic to the nilpotent Lie algebra $\mathfrak{n}_{2}$ and the $\mathrm{SU}(3)$-structure is the coupled one described before. If $b=0$, then $\mathfrak{s}_{a, 0}$ is solvable nonnilpotent, the $\mathrm{SU}(3)$-structure satisfies $\hat{d} \omega=0, \hat{d} \psi=0=\hat{d} \widehat{\psi}$, and the associated inner product $g=\sum_{k=1}^{6}\left(e^{k}\right)^{2}$ is flat.

From now on, we assume that $a \neq 0$ and $b \neq 0$. Under this assumption, the Lie algebra is solvable non-nilpotent and the $\mathrm{SU}(3)$-structure is coupled with

$$
\hat{d} \omega=b \psi
$$

Moreover, it has $w_{2}=-\frac{4}{3} b e^{12}+\frac{8}{3} b e^{34}-\frac{4}{3} b e^{56}$, which is easily seen to satisfy the condition $\hat{d} w_{2}=\frac{8}{3} b^{2} \psi$.

There exists a one-parameter family of derivations $D_{k} \in \operatorname{Der}\left(\mathfrak{s}_{a, b}\right)$ for which the 3 -form $\varphi=\omega \wedge \eta+\psi$ defines a closed $\mathrm{G}_{2}$-structure on the rank-one extension $\mathfrak{g}_{a, b, k}:=\mathfrak{s}_{a, b} \rtimes_{D_{k}} \mathbb{R}$. 
It has the following expression

$$
D_{k}=\left(\begin{array}{cccccc}
-\frac{b}{2} & k & 0 & 0 & 0 & 0 \\
-k & -\frac{b}{2} & 0 & 0 & 0 & 0 \\
0 & 0 & -\frac{b}{2} & -k & 0 & 0 \\
0 & 0 & k & -\frac{b}{2} & 0 & 0 \\
0 & 0 & 0 & 0 & 0 & 0 \\
0 & 0 & 0 & 0 & 0 & 0
\end{array}\right), \quad k \in \mathbb{R}
$$

and it is easily seen to satisfy the equation $D_{k}^{*} \psi=-b \psi$, for any $k \in \mathbb{R}$.

\section{Some Remarks on Closed $\mathrm{G}_{2}$-STRuCtures induCED By COUPled SU(3)-STRUCTURES ON LiE Algebras}

An interesting property of Lauret's example [37, Ex. 4.10], reviewed in Section 4.4, is that the left-invariant closed $\mathrm{G}_{2}$-structure induced by $\varphi=\omega \wedge \eta+\psi$ on the simply connected Lie group $\mathrm{G}_{a}$ is always a Laplacian soliton, that is, its Hodge Laplacian $\Delta_{\varphi} \varphi=-d *_{\varphi} d *_{\varphi} \varphi=d \tau$ satisfies the equation

$$
\Delta_{\varphi} \varphi=\lambda \varphi+\mathcal{L}_{X} \varphi
$$

for $\lambda=\lambda(a):=8 a^{2}-4 a-4$, and for a suitable vector field $X$ on $\mathrm{G}_{a}$. Moreover, this Laplacian soliton is algebraic according to [36, Def. 3.9], namely there exists a derivation $B \in \operatorname{Der}\left(\mathfrak{g}_{a}\right)$ such that the vector field $X$ is induced by the unique one-parameter group of automorphisms $\Phi_{t} \in \operatorname{Aut}\left(\mathrm{G}_{a}\right)$ satisfying $\left.d \Phi_{t}\right|_{1_{\mathrm{G}_{a}}}=\exp (t B) \in \operatorname{Aut}\left(\mathfrak{g}_{a}\right)$. Finally, depending on the value of $a$, the Laplacian soliton can be shrinking $(\lambda(a)<0)$, steady $(\lambda(a)=0)$, or expanding $(\lambda(a)>0)$.

\section{Remark 5.1.}

(1) So far, the shrinking Laplacian solitons on $\mathrm{G}_{a}$, for $\frac{1}{4} \leq a<1$, constitute the only known examples of such solitons.

(2) The left-invariant steady Laplacian soliton, corresponding to the value $a=1$, is also an ERP $\mathrm{G}_{2}$-structure. More generally, Lauret and Nicolini proved that every left-invariant ERP $\mathrm{G}_{2}$-structure on a simply connected Lie group is a steady Laplacian soliton [38]. On the other hand, there exists an example of a left-invariant steady Laplacian soliton that is not ERP [25].

On a (compact) 7-manifold $M$, all closed $\mathrm{G}_{2}$-structures satisfying (5.1) have a distinguished behaviour when they evolve under the $\mathrm{G}_{2}$-Laplacian flow. This flow, which was introduced by Bryant in [3], prescribes the evolution of a closed $\mathrm{G}_{2}$-structure $\varphi \in \Omega_{+}^{3}(M)$ as follows

$$
\left\{\begin{array}{l}
\frac{\partial}{\partial t} \varphi(t)=\Delta_{\varphi(t)} \varphi(t), \\
d \varphi(t)=0, \\
\varphi(0)=\varphi .
\end{array}\right.
$$

The initial datum $\varphi$ is a Laplacian soliton, i.e., it satisfies (5.1) for some real number $\lambda$ and some vector field $X \in \Gamma(T M)$, if and only if the solution $\varphi(t)$ of (5.2) starting from it at 
$t=0$ is self-similar, that is

$$
\varphi(t)=\left(1+\frac{2}{3} \lambda t\right)^{\frac{3}{2}} F_{t}^{*} \varphi,
$$

where $F_{t} \in \operatorname{Diff}(M)$ is the one-parameter family of diffeomorphisms generated by the vector field $X(t)=\left(1+\frac{2}{3} \lambda t\right)^{-1} X$ and satisfying $F_{0}=\operatorname{Id}_{M}$. In particular, $\varphi(t)$ exists on the maximal time interval $\left(-\infty,-\frac{3}{2 \lambda}\right)$ when $\lambda<0,(-\infty,+\infty)$ when $\lambda=0$, and $\left(-\frac{3}{2 \lambda},+\infty\right)$ when $\lambda>0$. We refer the reader to [3, 41] for more details on the $\mathrm{G}_{2}$-Laplacian flow and its solitons.

In [37, Lauret computed the solution of the $\mathrm{G}_{2}$-Laplacian flow on $\mathfrak{g}_{a}=\mathfrak{n}_{2} \rtimes_{D_{a}} \mathbb{R}$ starting from the closed $\mathrm{G}_{2}$-structure $\varphi=\omega \wedge \eta+\psi$. When $a \neq 1$, it is given by

$$
\varphi(t)=A_{t}^{q_{1}(a)} e^{127}+A_{t}^{q_{2}(a)} e^{347}+A_{t}^{q_{3}(a)}\left(e^{567}+e^{135}-e^{146}-e^{236}-e^{245}\right),
$$

where $e^{7}:=\eta, A_{t}=\frac{2}{3} \lambda(a) t+1$, and

$$
q_{1}(a)=\frac{3 a}{2(2 a+1)}, \quad q_{2}(a)=\frac{3(2 a-1)}{8(a-1)}, \quad q_{3}(a)=\frac{9}{8(2 a+1)(a-1)} .
$$

The maximal interval of existence of $\varphi(t)$ is $\left(-\infty,-\frac{3}{2 \lambda(a)}\right)$, when $\frac{1}{4} \leq a<1$, and $\left(-\frac{3}{2 \lambda(a)},+\infty\right)$, when $a>1$.

An examination of the solution $\varphi(t)$ allows us to notice the following remarkable property.

Proposition 5.2. Consider the seven-dimensional Lie algebra $\mathfrak{g}_{a}=\mathfrak{n}_{2} \rtimes_{D_{a}} \mathbb{R}$, for $a \geq \frac{1}{4}$ with $a \neq 1$, and let $\varphi=\omega \wedge \eta+\psi$ be the closed $\mathrm{G}_{2}$-structure on it induced by the coupled $\mathrm{SU}(3)$-structure $(\omega, \psi)$ on $\mathfrak{n}_{2}$ given in (4.5). Then, the solution of the $\mathrm{G}_{2}$-Laplacian flow starting from $\varphi$ at $t=0$ can be written as follows

$$
\varphi(t)=\omega(t) \wedge f(t) \eta+\psi(t)
$$

where $f(t)=\left(A_{t}\right)^{\frac{1}{2}}$ and the pair

$$
\begin{aligned}
& \omega(t)=A_{t}^{q_{1}(a)-\frac{1}{2}} e^{127}+A_{t}^{q_{2}(a)-\frac{1}{2}} e^{347}+A_{t}^{q_{3}(a)-\frac{1}{2}} e^{567}, \\
& \psi(t)=A_{t}{ }^{q_{3}(a)}\left(e^{135}-e^{146}-e^{236}-e^{245}\right)=A_{t}^{q_{3}(a)} \psi,
\end{aligned}
$$

defines a family of coupled $\mathrm{SU}(3)$-structures on $\mathfrak{n}_{2}$ satisfying

$$
\hat{d} \omega(t)=c(t) \psi(t), \quad c(t)=-\left(A_{t}\right)^{-\frac{1}{2}},
$$

and having $\hat{d} w_{2}(t)$ proportional to $\psi(t)$. Finally, $\varphi(t)$ induces the metric $g_{\varphi}(t)=g(t)+$ $[f(t)]^{2} \eta^{2}$, where $g(t)$ denotes the metric associated with the coupled $\mathrm{SU}(3)$-structure $(\omega(t), \psi(t))$.

Let $\mathfrak{h}$ denote a six-dimensional Lie algebra endowed with a coupled $\mathrm{SU}(3)$-structure $(\omega, \psi)$ satisfying $\hat{d} \omega=c \psi$, and assume that there exists a derivation $D \in \operatorname{Der}(\mathfrak{h})$ such that $D^{*} \psi=$ $-c \psi$. The observations given in Proposition 5.2 lead to the question whether the $\mathrm{G}_{2^{-}}$ Laplacian flow on $\mathfrak{h} \rtimes_{D} \mathbb{R}$ starting from $\varphi=\omega \wedge \eta+\psi$ can be reinterpreted as a flow on $\mathfrak{h}$ evolving the coupled SU(3)-structure $(\omega, \psi)$. The examination of the flow on the Lie algebras $\mathfrak{g}_{a, b}$ and $\mathfrak{g}_{a, b, k}$ defined in Section 4.4 may give some insights on this problem. For the sake of convenience, henceforth we let $e^{7}:=\eta$ and $e_{7}:=\xi$, so that the closed $\mathrm{G}_{2}$-structure $\varphi$ on 
these Lie algebras can be written as in (2.1) with respect to the basis $\left\{e^{1}, \ldots, e^{6}, e^{7}\right\}$ we are considering.

Let us begin with the Lie algebra $\mathfrak{g}_{a, b, k}$. Notice that the coupled $\mathrm{SU}(3)$-structure $(\omega, \psi)$ on $\mathfrak{s}_{a, b}$ satisfies the additional property $\hat{d} w_{2} \propto \psi$, which is also satisfied by the coupled $\mathrm{SU}(3)$-structure considered on $\mathfrak{n}_{2}$ and is never satisfied by any coupled $\mathrm{SU}(3)$-structure on $\mathfrak{n}_{1}$ (cf. Remark 4.5).

We consider the following Ansatz for the solution

$$
\varphi(t)=C_{1} e^{127}+C_{2} e^{347}+C_{3} e^{567}+C_{4} e^{135}-C_{5} e^{146}-C_{6} e^{236}-C_{7} e^{245},
$$

where $C_{i}=C_{i}(t), i=1, \ldots, 7$, are unknown positive functions depending only on $t$ and satisfying $C_{i}(0)=1$. Then, $\varphi(t)$ still defines a $\mathrm{G}_{2}$-structure, and it is closed if and only if $C_{7}=C_{6}=C_{5}=C_{4}=C_{2}$. Now, some straightforward computations show that the intrinsic torsion form $\tau(t)$ of $\varphi(t)$ has the following expression

$$
\tau(t)=-b\left(\frac{C_{1} C_{2}^{2}}{C_{3}^{2}}\right)^{\frac{1}{3}} e^{12}+3 b\left(\frac{C_{2}{ }^{5}}{C_{1}{ }^{2} C_{3}^{2}}\right)^{\frac{1}{3}} e^{34}-2 b\left(\frac{C_{2}{ }^{2} C_{3}}{C_{1}{ }^{2}}\right)^{\frac{1}{3}} e^{56} .
$$

Consequently, recalling that $\Delta_{\varphi(t)} \varphi(t)=d \tau(t)$, we have

$$
\Delta_{\varphi(t)} \varphi(t)=-b^{2}\left(\frac{C_{1} C_{2}^{2}}{C_{3}{ }^{2}}\right)^{\frac{1}{3}} e^{127}+3 b^{2}\left(\frac{C_{2}{ }^{5}}{C_{1}{ }^{2} C_{3}^{2}}\right)^{\frac{1}{3}}\left(e^{347}+e^{135}-e^{146}-e^{236}-e^{245}\right),
$$

whence we see that the Laplacian flow equation (5.2) is equivalent to the following system of ODEs for the functions $C_{1}, C_{2}, C_{3}$ :

$$
\left\{\begin{array}{l}
\frac{d}{d t} C_{1}=-b^{2}\left(\frac{C_{1} C_{2}^{2}}{C_{3}^{2}}\right)^{\frac{1}{3}} \\
\frac{d}{d t} C_{2}=3 b^{2}\left(\frac{C_{2}{ }^{5}}{C_{1}^{2} C_{3}^{2}}\right)^{\frac{1}{3}} \\
\frac{d}{d t} C_{3}=0
\end{array}\right.
$$

Combining these ODEs with the initial conditions $C_{1}(0)=1=C_{2}(0)=C_{3}(0)$, we immediately get $C_{3}(t)=1$ and $C_{1}=C_{2}^{-\frac{1}{3}}$, with $C_{2}$ solving the Cauchy problem

$$
\left\{\begin{array}{l}
\frac{d}{d t} C_{2}=3 b^{2} C_{2} \frac{17}{9} \\
C_{2}(0)=1
\end{array}\right.
$$

Thus, we have $C_{2}(t)=\left(-\frac{8}{3} b^{2} t+1\right)^{-\frac{9}{8}}$, and we see that the solution of the $\mathrm{G}_{2}$-Laplacian flow starting from $\varphi$ is defined in the interval $\left(-\infty, \frac{3}{8 b^{2}}\right)$. Moreover, it can be written as

$$
\varphi(t)=\omega(t) \wedge f(t) \eta+\psi(t)
$$

where $f(t)=\left(-\frac{8}{3} b^{2} t+1\right)^{\frac{1}{2}}$, and $(\omega(t), \psi(t))$ is a family of coupled $\mathrm{SU}(3)$-structures on $\mathfrak{s}_{a, b}$ satisfying $\hat{d} \omega(t)=c(t) \psi(t)$ with $c(t)=b\left(-\frac{8}{3} b^{2} t+1\right)^{-\frac{1}{2}}$ and having $\hat{d} w_{2}(t)$ proportional to $\psi(t)$.

Comparing this discussion with Proposition [5.2, we see that the solution we have just found shares similar properties with the solution of the Laplacian flow obtained by Lauret on $\mathfrak{g}_{a}$, for $\frac{1}{4} \leq a<1$. However, we claim that the closed $\mathrm{G}_{2}$-structure we are considering 
is never an algebraic soliton. The proof goes as follows. From [36], we know that if $\varphi$ gives rise to a left-invariant algebraic soliton on the simply connected Lie group with Lie algebra $\mathfrak{g}_{a, b, k}$, then there exist a derivation $B \in \operatorname{Der}\left(\mathfrak{g}_{a, b, k}\right)$ and a real number $\lambda$ such that

$$
\Delta_{\varphi} \varphi=d \tau=\lambda \varphi+B^{*} \varphi .
$$

Since $a \neq 0$ and $b \neq 0$, the space $\operatorname{Der}\left(\mathfrak{g}_{a, b, k}\right)$ is eight-dimensional, and we can write a generic derivation with respect to a basis $\left\{B_{1}, \ldots, B_{8}\right\}$ of $\operatorname{Der}\left(\mathfrak{g}_{a, b, k}\right)$ as $B=\sum_{i=1}^{8} A^{i} B_{i}$, for some real parameters $A_{i}$. We can then compute the 3 -form $B^{*} \varphi$, and see that the above equation reduces to a system of polynomial equations in the unknowns $A_{i}$ and $\lambda$. It turns out that this system has no solutions under the assumption $b \neq 0$.

On the other hand, obtaining the explicit solution of the $\mathrm{G}_{2}$-Laplacian flow on $\mathfrak{g}_{a, b}$ satisfying $\varphi(0)=\varphi$ seems challenging. For instance, the Ansatz (5.3) is not helpful in this case, as the flow equation in (5.2) implies that some of the functions $C_{i}(t)$ must be identically vanishing, a contradiction. Notice that in this example the coupled SU(3)-structure does not satisfy the condition $\hat{d} w_{2} \propto \psi$.

We now collect some observations and open problems motivated by the results presented in this last section.

(1) Is it possible to determine the explicit expression of the solution of the $\mathrm{G}_{2}$-Laplacian flow on $\mathfrak{g}_{a, b}$ starting from the closed $\mathrm{G}_{2}$-structure $\varphi$ ?

(2) Albeit the Lie algebras $\mathfrak{g}_{a}, \frac{1}{4} \leq a<1$, and $\mathfrak{g}_{a, b, k}, a \neq 0, b \neq 0$, are not isomorphic, they admit coupled SU(3)-structures sharing similar properties. Are the corresponding simply connected Lie groups endowed with the left-invariant coupled SU(3)-structures equivalent in a suitable sense?

(3) Consider a six-dimensional Lie algebra $\mathfrak{h}$ endowed with a coupled $\mathrm{SU}(3)$-structure $(\omega, \psi)$ with $\hat{d} \omega=c \psi$ and admitting a derivation satisfying $D^{*} \psi=-c \psi$. Is it possible to reinterpret the $\mathrm{G}_{2}$-Laplacian flow on $\mathfrak{g}=\mathfrak{h} \rtimes_{D} \mathbb{R}$ evolving the closed $\mathrm{G}_{2}$-structure $\varphi=\omega \wedge \eta+\psi$ as a flow evolving the coupled $\mathrm{SU}(3)$-structure on $\mathfrak{h}$ ? Does the condition $\hat{d} w_{2} \propto \psi$ play some role in this problem?

(4) Is it possible to classify all six-dimensional solvable Lie algebras admitting a coupled $\mathrm{SU}(3)$-structure up to isomorphism, at least when the condition $\hat{d} w_{2} \propto \psi$ holds?

Acknowledgements. The authors were supported by G.N.S.A.G.A. of I.N.d.A.M. and by the project P.R.I.N. 2017 "Real and Complex Manifolds: Topology, Geometry and Holomorphic Dynamics". A. F. would like to thank the organisers of the Abel Symposium, J. Figueroa-O'Farrill, S. Hervik, B. Kruglikov, I. Markina, J. Slovák, D. The, and B. Ørsted, for the wonderful conference and their hospitality.

\section{REFERENCES}

[1] Ball, G.: Seven-Dimensional Geometries With Special Torsion. Ph.D. dissertation, Duke University.

[2] Bonan, E.: Sur des variétés riemanniennes á groupe d'holonomie $\mathrm{G}_{2}$ ou $\operatorname{Spin}(7)$. C. R. Acad. Sci. Paris Sér. A-B 262, A127-A129 (1966).

[3] Bryant, R.: Some remarks on $\mathrm{G}_{2}$-structures. Proceedings of Gökova Geometry-Topology Conference 2005, Gökova Geometry/Topology Conference (GGT), Gökova, pp. 75-109 (2006).

[4] Chu, B. Y. : Symplectic homogeneous spaces. Trans. Amer. Math. Soc. 197, 145-159 (1974). 
[5] Conti, D., Fernández, M.: Nilmanifolds with a calibrated $G_{2}$-structure. Differ. Geom. Appl. 29 (4), 493-506 (2011).

[6] Corti, A., Haskins, M., Nordström, J., Pacini, T.: G G $_{2}$ manifolds and associative submanifolds via semiFano 3-folds. Duke Math. J. 164 (10), 1971-2092, (2015).

[7] Cleyton, R., Ivanov, S.: On the geometry of closed $\mathrm{G}_{2}$-structures. Comm. Math. Phys. 270 (1), 53-67 (2007)

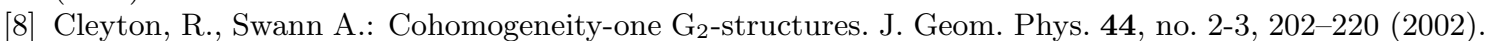

[9] Crowley, D., Nordström, J.: New invariants of $\mathrm{G}_{2}$-structures. Geom. Topol. 19 (5), 2949-2992 (2015).

[10] Diatta, A., Manga, B.: On properties of principal elements of Frobenius Lie algebras. J. Lie Theory 24 (3), 849-864 (2014)

[11] Fernández, M.: An example of a compact calibrated manifold associated with the exceptional Lie group $\mathrm{G}_{2}$. J. Differ. Geom. 26(2), 367-370 (1987).

[12] Fernández, M.: A family of compact solvable $\mathrm{G}_{2}$-calibrated manifolds. Tohoku Math. J. 39 (2), 287-289 (1987).

[13] Fernández, M., Fino, A., Kovalev, A., Munoz, V.: A compact $G_{2}$-calibrated manifold with first Betti number $b_{1}=1$. arXiv:1808.07144

[14] Fernández, M., Fino, A., Manero, V.: G ${ }_{2}$-structures on Einstein solvmanifolds, Asian J. Math. 19 (2), 321-342 (2015).

[15] Fernández, M., Fino, A., Raffero, A.: Locally conformal calibrated G 2 -manifolds. Ann. Mat. Pura Appl. 195 (5), 1721-1736 (2016).

[16] Fernández, M., Fino, A., Raffero, A.: Exact $\mathrm{G}_{2}$-structures on unimodular Lie algebras. To appear in Monatsh. Math. doi:10.1007/s00605-020-01429-0.

[17] Fernández, M., Gray, A.: Riemannian manifolds with structure group $G_{2}$. Ann. Mat. Pura Appl. 32, 19-45 (1982).

[18] Fernández, M., Manero, V., Otal, A., Ugarte, L.: Symplectic half-flat solvmanifolds. Ann. Global Anal. Geom. 43 (4), 367-383 (2013).

[19] Fine, J., Yao, C.: Hypersymplectic 4-manifolds, the $\mathrm{G}_{2}$-Laplacian flow, and extension assuming bounded scalar curvature, Duke Math. J. 167, 3533-3589 (2018).

[20] Fino, A., Raffero, A.: Coupled SU(3)-structures and supersymmetry. Symmetry 7 (2), 625-650 (2015).

[21] Fino, A., Raffero, A.: Einstein locally conformal calibrated G ${ }_{2}$-structures. Math. Z. 280 (3-4), 10931106 (2015).

[22] Fino, A., Raffero, A.: Closed $\mathrm{G}_{2}$-structures on non-solvable Lie groups. Rev. Mat. Complut. 32, no. 3, 837-851 (2019).

[23] Fino, A., Raffero, A.: Closed warped $\mathrm{G}_{2}$-structures evolving under the Laplacian flow. Ann. Sc. Norm. Sup. Pisa Cl. Sci. 20 (1), 315-348 (2020).

[24] Fino, A., Raffero, A.: A class of eternal solutions to the $\mathrm{G}_{2}$-Laplacian flow. arXiv:1807.01128 To appear in J. Geom. Anal.

[25] Fino, A., Raffero, A.: Remarks on homogeneous solitons of the $\mathrm{G}_{2}$-Laplacian flow. arXiv:1905.13078. To appear in C. R. Math. Acad. Sci. Paris.

[26] Freibert M.: Calibrated and parallel structures on almost Abelian Lie algebras. arXiv:1307.2542

[27] Gray, A.: Vector cross products on manifolds. Trans. Amer. Math. Soc. 141, 465-504 (1969).

[28] Harvey R., Lawson, H. B. Jr. : Calibrated geometries. Acta Math. 148, 47-157 (1982).

[29] Hitchin, N.: Stable forms and special metrics. In Global differential geometry: the mathematical legacy of Alfred Gray (Bilbao, 2000), volume 288 of Contemp. Math., pages 70-89. Amer. Math. Soc., Providence, RI (2001).

[30] Joyce, D. D.: Compact Riemannian 7-manifolds with holonomy G 2 . I, II. J. Differ. Geom. 43, 291-328, 329-375 (1996).

[31] Joyce, D. D.: Riemannian holonomy groups and calibrated geometry, volume 12 of Oxford Graduate Texts in Mathematics. Oxford University Press, Oxford, 2007.

[32] Joyce D. D., Karigiannis, S.: A new construction of compact torsion-free $\mathrm{G}_{2}$-manifolds by gluing families of Eguchi-Hanson spaces. arXiv:1707.09325. To appear in J. Differ. Geom.

[33] Kath, I., Lauret, J.: A new example of a compact ERP $\mathrm{G}_{2}$-structure. arXiv:2005.02462. 
[34] Kovalev, A.: Twisted connected sums and special Riemannian holonomy. J. Reine Angew. Math. 565, 125-160 (2003).

[35] Kovalev, A., Lee, N.-H.: K3 surfaces with non-symplectic involution and compact irreducible $\mathrm{G}_{2^{-}}$ manifolds. Math. Proc. Cambridge Philos. Soc. 151, 193-218 (2011).

[36] Lauret, J.: Laplacian flow of homogeneous $\mathrm{G}_{2}$-structures and its solitons. Proc. Lond. Math. Soc. 114 (3), 527-560 (2017).

[37] Lauret, J.: Laplacian solitons: questions and homogeneous examples. Differ. Geom. Appl. 54 (B), 345-360 (2017).

[38] Lauret, J., Nicolini, M.: Extremally Ricci pinched $\mathrm{G}_{2}$-structures on Lie groups. arXiv:1902.06375 To appear in Comm. Anal. Geom.

[39] Lauret, J., Nicolini, M.: The classification of ERP $\mathrm{G}_{2}$-structures on Lie groups. To appear in Ann. Mat. Pura Appl. doi:10.1007/s10231-020-00977-4.

[40] Lichnerowicz, A., Medina, A.: On Lie groups with left-invariant symplectic or Kählerian structures. Lett. Math. Phys. 16 (3), 225-235 (1988).

[41] Lotay, J. D., Wei, Y.: Laplacian flow for closed $\mathrm{G}_{2}$ structures: Shi-type estimates, uniqueness and compactness. Geom. Funct. Anal. 27 (1), 165-233 (2017).

[42] Manero, V.: Compact solvmanifolds with calibrated and cocalibrated $\mathrm{G}_{2}$-structures. Manuscripta Math. 162, 315-339 (2020).

[43] Podestà, F., Raffero, A.: On the automorphism group of a closed $\mathrm{G}_{2}$-structure. Q. J. Math. 70 (1), 195-200 (2019).

[44] Podestà, F., Raffero, A.: Homogeneous symplectic half-flat 6-manifolds. Ann. Global Anal. Geom. 55 (1), 1-15 (2019)

[45] Podestà, F., Raffero, A.: On the automorphism group of a symplectic half-flat 6-manifold. Forum Math. 31 (1), 265-273 (2019).

[46] Podestà, F., Raffero, A.: Closed $\mathrm{G}_{2}$-structures with a transitive reductive group of automorphisms. arXiv:1911.13052

Dipartimento di Matematica "G. Peano", Università degli Studi di Torino, Via Carlo AlBERTO 10, 10123 TORINO, ITALY

E-mail address: annamaria.fino@unito.it

Dipartimento di Matematica "G. Peano", Università degli Studi di Torino, Via Carlo AlBERTO 10, 10123 TORINO, ITALY

E-mail address: alberto.raffero@unito.it 